\title{
Endoscopic Adhesiolysis of Flexor Hallucis Longus Muscle
}

\author{
Tun Hing Lui, M.B.B.S.(HK), F.R.C.S.(Edin), F.H.K.A.M., F.H.K.C.O.S.
}

\begin{abstract}
Adhesion of the flexor hallucis longus (FHL) muscle to the distal tibia can occur after distal tibial fracture, distal fibular fracture, low tibial osteotomy, soft-tissue injury at the posterior ankle, subclinical compartment syndrome of the distal deep posterior compartment of the leg, or Volkmann contracture after deep posterior compartment syndrome of the leg. The purpose of this Technical Note is to report the endoscopic approach of FHL muscle adhesiolysis. It is indicated in patients with symptomatic adhesion of the FHL muscle and contraindicated if there is entrapment of the FHL muscle or tendon in the fracture callus or if there is extensive fibrosis and contracture of the FHL muscle as a result of Volkmann contracture after deep posterior compartment syndrome of the leg.
\end{abstract}

A dhesion of the flexor hallucis longus (FHL) muscle to the distal tibia can occur after distal tibial fracture, $^{1,2}$ distal fibular fracture, ${ }^{3}$ low tibial osteotomy, soft-tissue injury at the posterior ankle, subclinical compartment syndrome of the distal deep posterior compartment of the leg, ${ }^{4-7}$ or Volkmann contracture after deep posterior compartment syndrome of the leg. The distal third and fourth portion of the FHL muscle is located in a more compressed and deeper compartment. ${ }^{7,8}$ A localized increase in pressure can result in ischemia of the distal part of the FHL muscle belly. Because only a small portion of the muscle is involved, there is no retraction of the main muscle belly. The necrotic part of the muscle can become fibrotic and adhere to the surrounding structures. Immobilization of the fibrotic portion during the posttrauma period can further reduce muscle and tendon mobility. ${ }^{4}$ The patient may complain of deep posteromedial ankle pain, checkrein deformity of the hallux, ${ }^{1,4}$ or hallux flexus. ${ }^{9,10}$ Progressive fibrosis at the FHL myotendinous junction can also result in limitation

From the Department of Orthopaedics and Traumatology, North District Hospital, Hong Kong, China.

The author reports that he has no conflicts of interest in the authorship and publication of this article.

Received September 4, 2016; accepted October 4, 2016.

Address correspondence to Tun Hing Lui, M.B.B.S.(HK), F.R.C.S.(Edin), F.H.K.A.M., F.H.K.C.O.S., Department of Orthopaedics and Traumatology, North District Hospital, 9 Po Kin Road, Sheung Shui, NT, Hong Kong SAR, China.E-mail: luithderek@yahoo.co.uk

(C) 2016 by the Arthroscopy Association of North America $2212-6287 / 16857 / \$ 36.00$

http://dx.doi.org/10.1016/j.eats.2016.10.006 of first metatarsophalangeal dorsiflexion and increased loading of the first metatarsophalangeal joint, thereby leading to hallux rigidus. ${ }^{11}$ Surgical release of the FHL muscle $^{4,9}$ or lengthening of the FHL tendon ${ }^{5,12}$ has been proposed. Classically, FHL release is performed with an open approach requiring a large incision with extensive soft-tissue dissection especially around the neurovascular structures. ${ }^{10}$

We describe an endoscopic approach for release of the FHL muscle from the distal tibia with the advantage of minimal soft-tissue dissection. ${ }^{13}$ It is indicated if there is symptomatic fibrous adhesion of the distal FHL muscle to the distal tibia as a result of distal tibial or fibular fracture, after low tibial osteotomy, or after distal deep posterior compartment syndrome. The localized involvement of the distal part of the FHL muscle after distal deep posterior compartment syndrome can explain why release of the FHL muscle and tendon without tendon lengthening can improve the condition. ${ }^{4}$ This minimally invasive approach is contraindicated if there is entrapment of the FHL muscle or tendon in the fracture callus ${ }^{1,10}$ or if there is extensive fibrosis and contracture of the FHL muscle as a result of Volkmann contracture after deep posterior compartment syndrome of the leg (Table 1). Resection of the callus or lengthening of the FHL tendon is more appropriate in these situations.

\section{Technique}

\section{Preoperative Assessment and Patient Positioning}

The location of pain should be carefully identified. The pain should be at the posterior part of the ankle 
Table 1. Indications and Contraindications of Endoscopic Adhesiolysis of FHL Muscle

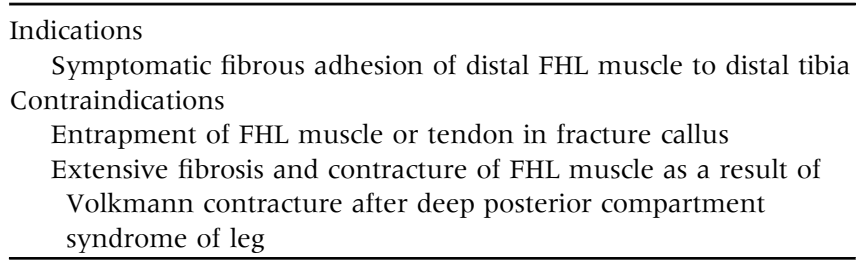

FHL, flexor hallucis longus.

joint or the distal tibia. There will be local tenderness corresponding to the site of pain. The proximal extent of tenderness should be noted because this guides the extent of release required. The range of ankle motion is determined and is usually comparable to that of the contralateral ankle. The dorsiflexion range of the first metatarsophalangeal joint is assessed with the ankle in plantar flexion and dorsiflexion. Any hallux flexus deformity with the ankle in dorsiflexion is recorded. Radiographs of the ankle are taken, and any bony prominence at the posterior surface of the distal tibia should be noted.

The patient is in the prone position with a thigh tourniquet to provide a bloodless operative field. A $4.0-\mathrm{mm} 30^{\circ}$ arthroscope (Dyonics; Smith \& Nephew, Andover, MA) is used for this procedure.

\section{Portal Placement}

Posterior ankle endoscopy ${ }^{14}$ is performed with the posteromedial and posterolateral portals. The posterolateral portal is located on the lateral side of the Achilles tendon just above the posterior calcaneal tubercle. The posteromedial portal is located at the intersection point between the medial border of the Achilles tendon and the line joining the undersurface of the sustentaculum tali and first metatarsal (Fig 1). ${ }^{15,16}$ Five-millimeter skin incisions are made at the portal sites, the subcutaneous tissue is bluntly dissected by a hemostat, and the investing fascia is penetrated by the tip of the hemostat.

\section{Identification of FHL Tendon}

The posterolateral portal is the viewing portal. The adipose tissue of the posterior ankle is debrided by an arthroscopic shaver (Dyonics; Smith \& Nephew) through the posteromedial portal, starting from the lateral side of the posterior ankle, until the FHL tendon is exposed. The FHL tendon can be confirmed as it moves with plantar flexion and dorsiflexion of the great toe.

\section{Endoscopic Release of Fascia Over FHL Muscle}

The posterolateral portal is the viewing portal. The FHL tendon is traced proximally to identify the FHL muscle. The fascia over the FHL muscle is released by an arthroscopic shaver and arthroscopic scissors (Arthrex, Naples, FL) through the posteromedial portal
(Fig 2). Release of the fascia should be proximal enough to allow subsequent release of the FHL muscle. The texture of the FHL muscle is assessed by an arthroscopic probe (Arthrex). The fibrosis may only be limited to the deep surface of the FHL muscle adherent to the distal tibia. If there is extensive fibrosis of the muscle, it may be due to Volkmann contracture of the muscle resulting from neglected posterior leg compartment syndrome. It is usually associated with hallux flexus deformity especially with ankle dorsiflexion. Lengthening of the FHL tendon together with release of the FHL muscle is indicated in this situation.

\section{Endoscopic Adhesiolysis of FHL Muscle}

The posterolateral portal is the viewing portal. The FHL muscle is released from the distal tibia with an arthroscopic shaver through the posteromedial portal. The release starts from lateral medially and from distal proximally. The cutting blade of the shaver should face toward the tibia, and suction should be kept to a minimum. This can reduce the extent of injury to the FHL muscle. The release should go proximally, passing the zone of fibrosis, until a normal muscle-bone interface is seen (Fig 3). The arthroscope is switched to the posteromedial portal. Release of the FHL muscle is performed by the arthroscopic shaver through the posterolateral portal. This starts from the medial edge of the muscle and goes laterally (Fig 4) until the previously laterally released muscle-bone interface is reached. Again, the release should go proximally

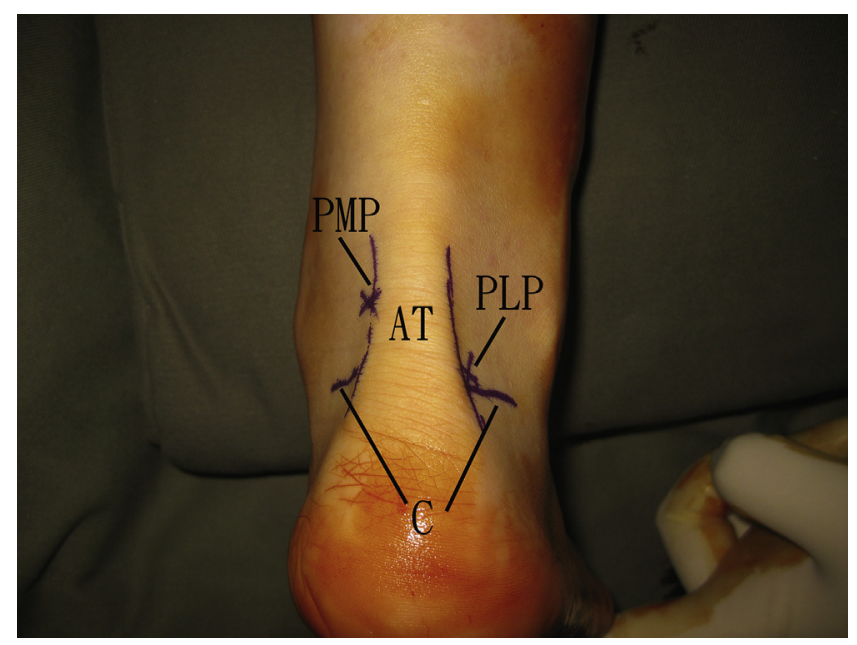

Fig 1. Endoscopic adhesiolysis of flexor hallucis longus muscle in a right ankle. The patient is in the prone position. Posterior ankle endoscopy is performed with the posteromedial and posterolateral portals. The posterolateral portal (PLP) is located on the lateral side of the Achilles tendon (AT) just above the posterior calcaneal tubercle $(\mathrm{C})$. The posteromedial portal (PMP) is located at the intersection point between the medial border of the Achilles tendon and the line joining the undersurface of the sustentaculum tali and first metatarsal. 
Fig 2. Endoscopic adhesiolysis of flexor hallucis longus muscle (FHLm) in a right ankle. (A) The patient is in the prone position. The posterolateral portal (PLP) is the viewing portal, and the posteromedial portal (PMP) is the working portal. (AT, Achilles tendon.) (B) The flexor hallucis longus tendon is traced proximally to identify the FHLm. The fascia (F) over the FHLm is released by an arthroscopic shaver and arthroscopic scissors through the posteromedial portal.

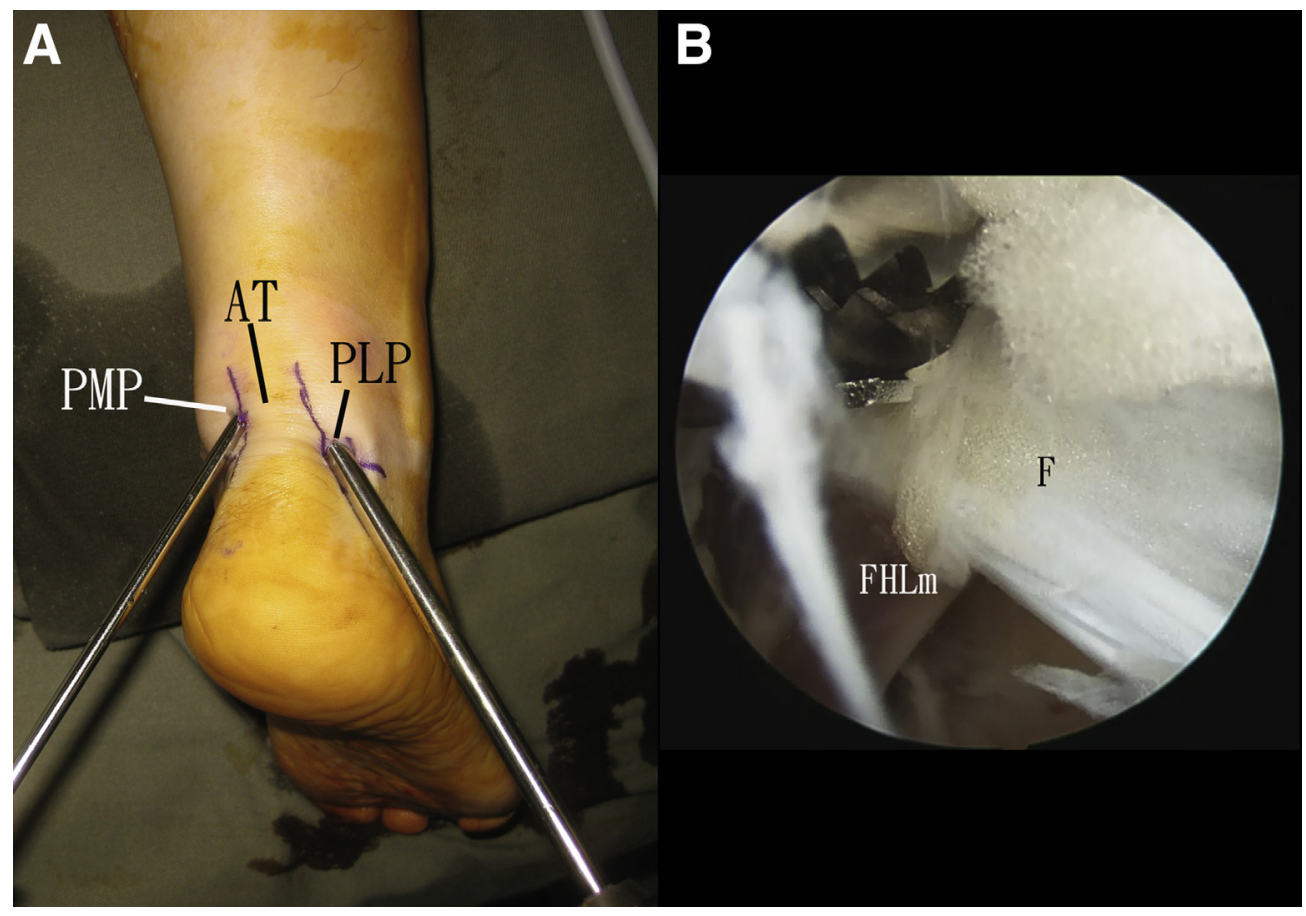

enough to pass the zone of fibrosis until the normal muscle-bone interface is seen (Fig 5). This ensures complete release of the FHL muscle from the distal tibia (Video 1). Any posterior tibial bony prominence, if

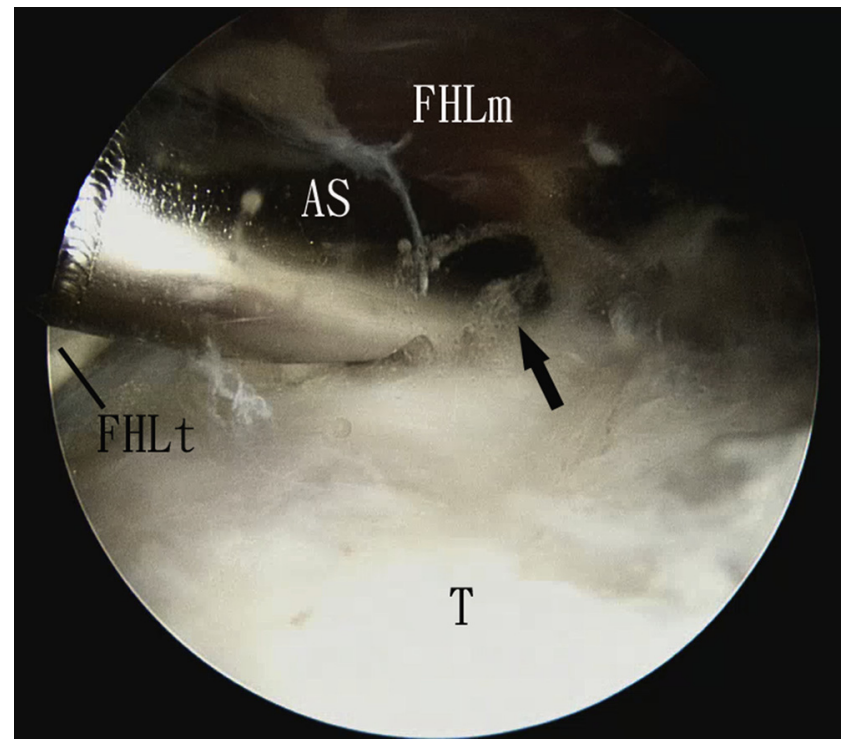

Fig 3. Endoscopic adhesiolysis of flexor hallucis longus muscle (FHLm) in a right ankle. The patient is in the prone position. The posterolateral portal is the viewing portal. The FHLm is released from the distal tibia (T) with an arthroscopic shaver (AS) through the posteromedial portal. The release starts from lateral medially and from distal proximally. The arrow indicates the direction of release of the FHLm from lateral medially. (FHLt, flexor hallucis longus tendon.) present, is resected with an arthroscopic acromionizer (Dyonics; Smith $\&$ Nephew). Active and passive mobilization of the ankle and the great toe can be started from postoperative day 1 .

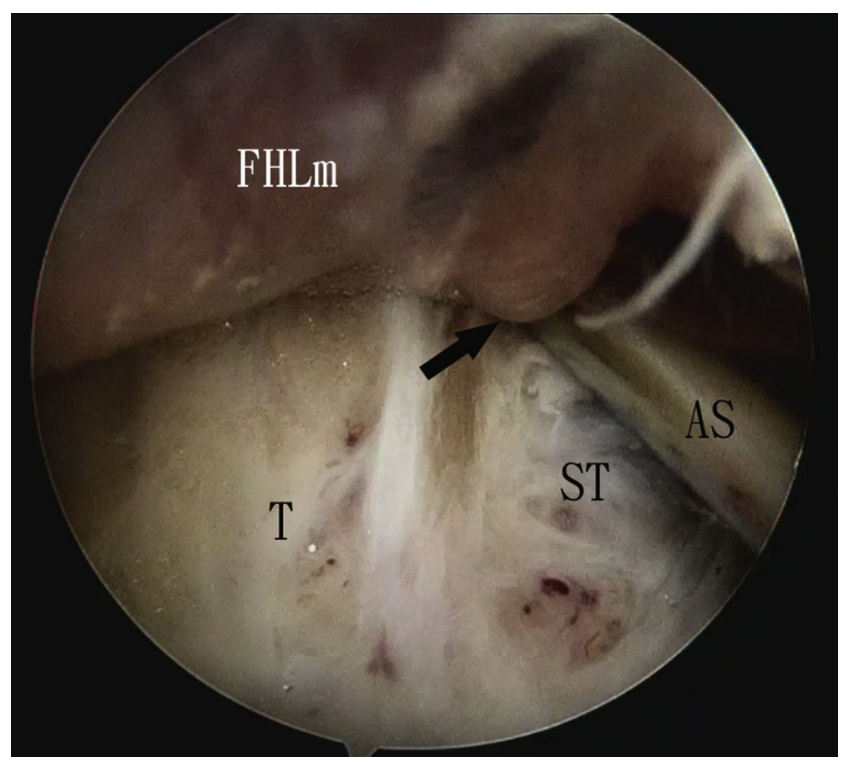

Fig 4. Endoscopic adhesiolysis of flexor hallucis longus muscle (FHLm) in a right ankle. The patient is in the prone position. The posteromedial portal is the viewing portal. Release of the FHLm is performed by the arthroscopic shaver (AS) through the posterolateral portal. It starts from the medial edge of the muscle and goes laterally. The arrow indicates the direction of release of the FHLm from medial laterally. (ST, scar tissue; $\mathrm{T}$, distal tibia.) 


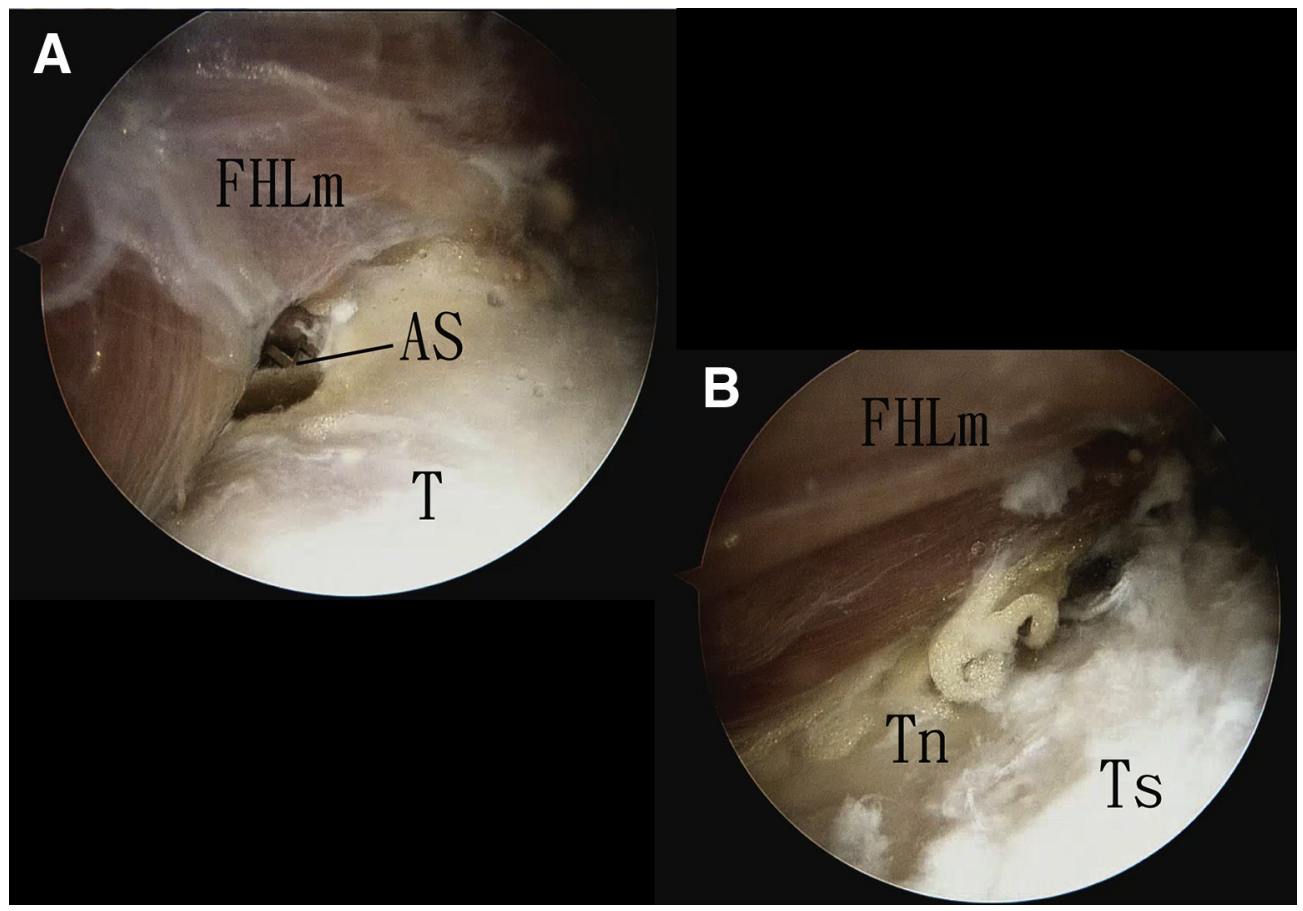

Fig 5. Endoscopic adhesiolysis of flexor hallucis longus muscle (FHLm) in a right ankle. The patient is in the prone position. The posterolateral portal is the viewing portal. The release is completed when the release is throughout the span of fibrosis in the medial-lateral and proximal-distal directions. (A) Complete release in the mediallateral direction is confirmed when the arthroscopic shaver (AS) from the medial side of the FHLm can pass between the muscle and distal tibia $(\mathrm{T})$ to the lateral side of the FHLm. (B) Complete release in the proximal-distal direction can be confirmed if the release passes through the zone of scarred tibia (Ts) and a normal distal tibia without scarring (Tn) is seen.

\section{Discussion}

Open FHL release can successfully deal with adhesion of the FHL muscle to the tibia at the cost of a large incision with extensive soft-tissue dissection especially around the neurovascular structures. With the advancements in hindfoot endoscopy, ${ }^{14,17}$ the procedure can be performed in a minimally invasive manner. Dissection of the neurovascular bundle is not needed to approach the FHL muscle during the endoscopic procedure. To avoid injury to the posterior tibial neurovascular bundle or its branches during adhesiolysis, the release should be started at the lateral border of the muscle and go medially. In this way, the neurovascular bundle will be protected by the FHL muscle. ${ }^{18}$ However, the release of the most medial part of the muscle may not be adequate by the

Table 2. Pearls and Pitfalls of Endoscopic Adhesiolysis of FHL Muscle

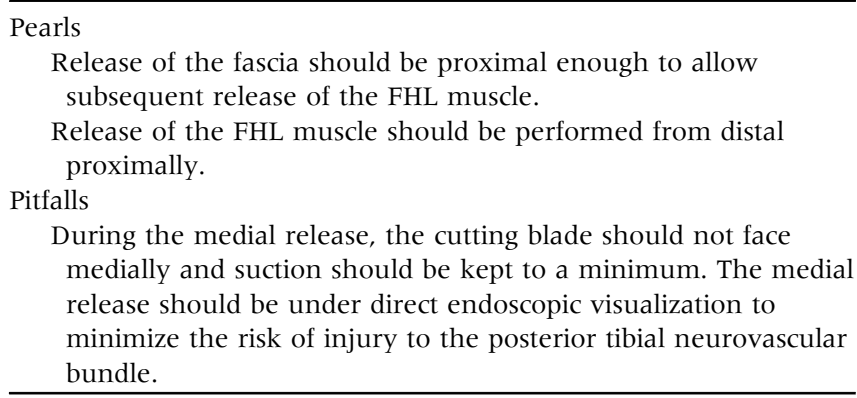

FHL, flexor hallucis longus. lateral approach. The release of the medial part of the muscle is performed at the medial border of the muscle. During the medial release, the cutting blade should not face medially and suction should be kept to a minimum. The medial release should be performed under direct endoscopic visualization. These precautions can minimize the risk of injury to the posterior tibial neurovascular bundle (Table 2 ).

The potential advantages of the described technique include smaller wounds and better cosmetic results, minimal soft-tissue dissection, and the allowance of early mobilization. The potential risks of this technique include injury to the FHL tendon and muscle, injury to the posterior tibial neurovascular bundle, inadequate muscle release, and adhesion recurrence (Table 3). This technique is not technically demanding and can be performed by the average foot and ankle arthroscopist.

Table 3. Advantages and Risks of Endoscopic Adhesiolysis of FHL Muscle

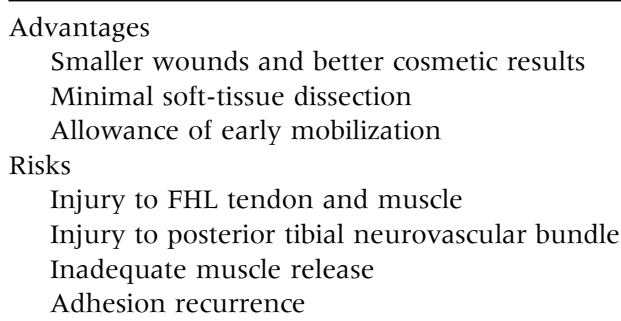

FHL, flexor hallucis longus. 


\section{References}

1. Rosenberg GA, Sferra JJ. Checkrein deformity-An unusual complication associated with a closed Salter-Harris type II ankle fracture: A case report. Foot Ankle Int 1999;20:591-594.

2. Berentey G, Tamasy S. Adhesion of the tendon of the m. flexor hallucis longus following tibial fracture. Magy Traumatol Orthop Helyreallito Seb 1973;16:161-168 [in Hungarian].

3. Leitschuh PH, Zimmerman JP, Uhorchak JM, Arciero RA, Bowser L. Hallux flexion deformity secondary to entrapment of the flexor hallucis longus tendon after fibular fracture. Foot Ankle Int 1995;16:232-235.

4. Fitoussi F, Ilharreborde B, Guerin F, Souchet P, Pennecot GF, Mazda K. Claw toes after tibial fracture in children. J Child Orthop 2009;3:339-343.

5. Hernandez-Cortes P, Pajares-Lopez M, HernandezHernandez MA. Ischemic contracture of deep posterior compartment of the leg following isolated ankle fracture. J Am Podiatr Med Assoc 2008;98:404-407.

6. Kwiatkowski TC, Detmer DE. Anatomical dissection of the deep posterior compartment and its correlation with clinical reports of chronic compartment syndrome involving the deep posterior compartment. Clin Anat 1997; 10:104-111.

7. Piper KJ, Yen-yi JC, Horsley M. Missed posterior deep, inferior subcompartment syndrome in a patient with an ankle fracture: A case report. J Foot Ankle Surg 2010;49: 398.e5-398.e8.

8. Sassu P, Acland RD, Salgado CJ, Mardini S, Ozyurekoglu T, Anatomy and vascularization of the flexor hallucis longus muscle and its implication in free fibula flap transfer. An anatomical study. Ann Plast Surg 2010;64:233-237.
9. Burda R, Morochovic R, Kitka M. Hallux flexus-The result of posttraumatic entrapment of the flexor hallucis longus tendon in the tibial fracture site. Rozhl Chir 2010;89:466-467 [in Slovak].

10. Lee HS, Kim JS, Park SS, Lee DH, Park JM, Wapner KL. Treatment of checkrein deformity of the hallux. J Bone Joint Surg Br 2008;90:1055-1058.

11. Kirane YM, Michelson JD, Sharkey NA. Contribution of the flexor hallucis longus to loading of the first metatarsal and first metatarsophalangeal joint. Foot Ankle Int 2008;29:367-377.

12. Yuen CP, Lui TH. Adhesion of flexor hallucis longus at the site of a tibial-shaft fracture-A cause of a checkrein deformity. Foot Ankle Surg 2015;21:e23-e26.

13. Lui TH. Endoscopic adhesiolysis of the flexor hallucis longus muscle. Foot Ankle Spec 2014;7:492-494.

14. van Dijk CN, Scholten PE, Krips R. A 2-portal endoscopic approach for diagnosis and treatment of posterior ankle pathology. Arthroscopy 2000;16:871-876.

15. Lui TH. Flexor hallucis longus tendoscopy: A technical note. Knee Surg Sports Traumatol Arthrosc 2009;17:107-110.

16. Lui TH. Endoscopic assisted flexor hallucis tendon transfer in the management of chronic rupture of Achilles tendon. Knee Surg Sports Traumatol Arthrosc 2007; 15:1163-1166.

17. Ogut T, Ayhan E, Irgit K, Sarikaya AI. Endoscopic treatment of posterior ankle pain. Knee Surg Sports Traumatol Arthrosc $2011 ; 19: 1355-1361$.

18. Apaydin N, Loukas M, Kendir S, et al. The precise localization of distal motor branches of the tibial nerve in the deep posterior compartment of the leg. Surg Radiol Anat 2008;30:291-295. 